\title{
High-Frequency Noise in Modern FET/HEMT Channels Caused by the Excitation of 2D-Plasma Waves
}

\author{
P. Shiktorov ${ }^{a}$, E. Starikov ${ }^{a}$, V. Gružinskis ${ }^{a}$, L. VArani ${ }^{b}$ And L. ReGGiani ${ }^{c}$ \\ ${ }^{a}$ Semiconductor Physics Institute, A. Goštauto 11, LT 01108 Vilnius, Lithuania \\ ${ }^{b}$ Institut d'Électronique du Sud (CNRS UMR 5214), Université Montpellier II \\ 34095 Montpellier Cedex 5, France \\ ${ }^{c}$ Dipartimento di Ingegneria dell'Innovazione and CNISM, Università del Salento \\ Via Arnesano s/n, 73100 Lecce, Italy
}

\begin{abstract}
The problems related with the intrinsic noise in FET/HEMT channels induced by continuous branching of the total current between channel and gate are considered in the framework of a simple analytical model and its predictions on the current-noise spectra. Main branching-induced effects such as the appearance of an additional noise related to the excitation of plasma waves, its dependence on FET/HEMT embedding circuits, interference properties, etc. are analysed.
\end{abstract}

PACS: $72.20 . \mathrm{Ht}, 72.30 .+\mathrm{q}, 72.70 .+\mathrm{m}$

\section{Introduction}

It is well known that the characteristics of the internal electronic noise of a device reflect sufficiently well the information related to both the eigenfrequency spectrum [1] and the state of the free carrier system and its changes (for example, in going from equilibrium to nonequilibrium conditions). Such a dependence of the internal noise characteristics on physical behavior of free carriers is often used as a precursor of the system under test transition from a first physical state to another one such as, for instance, the transition from a static to a dynamic state, onset of generation processes, etc. [2, 3].

At the present time, one of the promising trends in ultrafast modern electronics is the development of $\mathrm{THz}$ range solid-state devices based on the usage of micro- and nanometer FET/HEMT structures for the resonant detection and generation of $\mathrm{THz}$ radiation. Mainly, this is caused by the possibility of easy tuning the $2 \mathrm{D}$ plasma excitation spectrum inside the transistor channel by changing the external conditions, namely: gate voltage, drain voltage, operation regime, etc. [1, 4, 5].

Usually, the most effective way to analyze the eigenfrequency spectrum is related with consideration of frequency behavior of the current and voltage noise spectra of a device under test. Therefore, investigations of intrinsic noise of modern FETs/HEMTs structures related to an excitation of $2 \mathrm{D}$ plasma waves is rather essential for their characterization for $\mathrm{THz}$ applications.

Usually, an electron transport and noise analysis is based on the conservation law of total current $\oint_{S} \boldsymbol{j}_{\text {tot }} \mathrm{d} \boldsymbol{S}=0$ where $\boldsymbol{j}_{\text {tot }}=\varepsilon \varepsilon_{0} \partial \boldsymbol{E} / \partial t+\boldsymbol{j}^{\text {drift }}$ is the lo- cal total current density consisting of the displacement and conduction (drift) components, and $\boldsymbol{S}$ is a surface surrounding some volume of interest. In the case of two-terminal devices (resistors or diodes), a one-dimensional treatment of carrier transport, which supposes that $\boldsymbol{j}^{\text {drift }}|| \boldsymbol{E}|| x$, is usually used. In this case the conservation law reduces to the scalar relation: $\frac{\partial}{\partial x}\left[S(x) j_{\text {tot }}(x, t)\right]=0$ which corresponds to the current flow conservation inside a tube with cross-section $S(x)$. In going from two- to three-terminal (transistors) devices the local parallelism of $\boldsymbol{j}^{\text {drift }}$ and $\boldsymbol{E}$ inside the device is in general violated, so that a one-dimensional approximation no longer applies. This implies an appearance of the branching of total current tubes, and as a result the total current conservation must be formulated as zero-sum rule for incoming/outcoming currents for a certain volume bounded by some closed surface $\boldsymbol{S}$. Such a situation is typical in FET/HEMT structures. Here, the conduction current $\boldsymbol{j}^{\text {drift }}$ mainly flows along a conducting channel while the governing direction of the local electric field $\boldsymbol{E}$ does not coincide with the current flow due to the presence of a gate. In essence, under the gate action, the whole channel becomes practically a continuous region of total-current branching between the source-drain and channel-gate directions. The aim of this report is to elucidate noise phenomena induced by such a branching.

\section{Analytical model}

Following Ref. [1], we shall describe the self-consistent distribution of charge density $\rho^{3 \mathrm{D}}(x)$ and potential $\varphi(x)$ in FETs/HEMTs channels by using the following system 
of equations which includes: (i) the charge conservation law

$$
\frac{\partial}{\partial t} \rho^{3 \mathrm{D}}(x)+\frac{\partial}{\partial x} j_{\mathrm{c}}^{\mathrm{drift}}(x)=0
$$

and (ii) the one-dimensional (1D) approximation for the two-dimensional (2D) Poisson equation

$$
\varepsilon_{\mathrm{c}} \frac{\partial^{2}}{\partial x^{2}} \varphi(x)+\frac{\varepsilon_{\mathrm{d}}}{d(x) \delta}\left[U_{\mathrm{g}}-\varphi(x)\right]=-\frac{1}{\varepsilon_{0}} \rho^{3 \mathrm{D}}(x),
$$

which takes into account the gate influence (second term in left hand side (1.h.s.) of Eq. (2)) on the potential distribution. Here, $U_{\mathrm{g}}$ is the gate potential, $d(x)$ is the local gate-to-channel distance, $\delta$ is the channel width, $\varepsilon_{\mathrm{c}}$ and $\varepsilon_{\mathrm{d}}$ are the dielectric constants in the channel and the gate-to-channel spacer, respectively.

To close Eqs. (1) and (2) we shall use the hydrodynamic approach to describe the current drift component along the channel, $j_{\mathrm{c}}^{\text {drift }}(x)=e n^{3 \mathrm{D}}(x) v(x)$ determined by the free electron stream with concentration $n^{3 \mathrm{D}}$ which flows upon the homogeneous donor background with concentration $N^{3 \mathrm{D}}$ and where the velocity of the electron flow is described as

$$
\begin{aligned}
& \frac{\partial}{\partial t} v+\frac{\partial}{\partial x}\left[\frac{v^{2}}{2}+\frac{e}{m^{*}} \varphi(x)\right]+e \nu D \frac{\partial}{\partial x} n^{3 \mathrm{D}}(x) \\
& =-\nu v+\tilde{f},
\end{aligned}
$$

where $m^{*}$ is the carrier effective mass, $\nu$ is the velocity relaxation rate, $D$ is the longitudinal diffusion coefficient, $\tilde{f}$ is the Langevin force with a spectral density $\delta$-correlated in space, $S_{f f}=4 k_{b} T \nu / m^{*}$. By linearizing Eqs. (1), (2) one can construct an analytical model which describes transient characteristics, impedance/admittance matrixes, Norton and Thevenin generators of diffusion noise, etc.

In the most interesting case of common-source configuration, where the leading role is played by current variations in the source-drain (SD) and source-gate (SG) circuits, $\Delta J_{\mathrm{d}}$ and $\Delta J_{\mathrm{g}}$, respectively, the fluctuations of currents and potentials in SD and SG circuits can be presented in a vector form as

$$
\Delta \boldsymbol{J}=\left[\begin{array}{c}
\Delta J_{\mathrm{d}} \\
\Delta J_{\mathrm{g}}
\end{array}\right], \quad \Delta \boldsymbol{U}=\left[\begin{array}{c}
\Delta U_{\mathrm{d}} \\
\Delta U_{\mathrm{g}}
\end{array}\right] .
$$

Here, the explicit linearized dependence of $\Delta \boldsymbol{J}$ on $\Delta \boldsymbol{U}$ and $\tilde{f}$ takes the form

$$
\Delta \boldsymbol{J}=\hat{Y} \Delta \boldsymbol{U}+\int_{0}^{L} \boldsymbol{G}_{J}(\omega) \tilde{f}\left(x_{0}\right) \mathrm{d} x_{0},
$$

where

$$
\hat{Y}=\varepsilon_{\mathrm{c}} \varepsilon_{0} \frac{\omega_{\mathrm{p}}^{2}}{\mathrm{i} \omega+\nu} \frac{\beta}{\alpha} \frac{\operatorname{ch} \beta L-1}{\operatorname{sh} \beta L}\left(\begin{array}{cc}
\frac{\operatorname{ch} \beta L}{\operatorname{ch} \beta L-1} & -1 \\
-1 & 2
\end{array}\right)
$$

is the matrix of HEMTs/FETs admittance under the common-source operation, and

$$
\boldsymbol{G}_{J}\left(\omega, x_{0}\right)=\varepsilon_{\mathrm{c}} \varepsilon_{0} \frac{\omega_{\mathrm{p}}^{2} \beta}{\mathrm{i} \omega+\nu}\left[\begin{array}{c}
\operatorname{ch} \beta x_{0} / \operatorname{sh} \beta L \\
\operatorname{sh} \beta\left(\frac{L}{2}-x_{0}\right) / \operatorname{ch} \beta \frac{L}{2}
\end{array}\right]
$$

is the response function in $\mathrm{SD}$ and $\mathrm{SG}$ circuits which determines in accordance with the Thevenin generator of noise (see below Eq. (11)), $\omega_{\mathrm{p}}=\sqrt{\mathrm{e}^{2} n / \varepsilon_{\mathrm{c}} \varepsilon_{0} m^{*}}$ is the volume plasma frequency of electrons in the channel, and $\alpha=\omega_{\mathrm{p}}^{2} /\left[\omega_{\mathrm{p}}^{2}+\mathrm{i} \omega(\mathrm{i} \omega+\nu)\right], \beta^{2}=\lambda^{2}\left[\mathrm{i} \omega(\mathrm{i} \omega+\nu) /\left(\omega_{\mathrm{p}}^{2}+\right.\right.$ $\mathrm{i} \omega(\mathrm{i} \omega+\nu))], L$ is the channel length, $\lambda=\sqrt{\left(\varepsilon_{\mathrm{d}} / \varepsilon_{\mathrm{c}}\right) / d \delta}$. By considering the impedance matrix,

$$
\begin{aligned}
\hat{Z} & =\hat{Y}^{-1}=\left(\varepsilon_{\mathrm{c}} \varepsilon_{0} \frac{\omega_{\mathrm{p}}^{2}}{\mathrm{i} \omega+\nu} \frac{\beta}{\alpha}\right)^{-1} \\
& \times \frac{\operatorname{sh} \beta L}{\operatorname{ch} \beta L+1}\left(\begin{array}{cc}
2 & 1 \\
1 & \frac{\operatorname{ch} \beta L}{\operatorname{ch} \beta L-1}
\end{array}\right)
\end{aligned}
$$

Eq. (5) can be rewritten as

$$
\Delta \boldsymbol{U}=\hat{Z} \Delta \boldsymbol{J}+\int_{0}^{L} \boldsymbol{G}_{\mathrm{U}}\left(\omega, x_{0}\right) \tilde{f}\left(x_{0}\right) \mathrm{d} x_{0},
$$

where

$$
\begin{aligned}
& \boldsymbol{G}_{\mathrm{U}}\left(\omega, x_{0}\right)=-\hat{Z} \boldsymbol{G}_{J}\left(\omega, x_{0}\right) \\
& =\alpha\left[\begin{array}{c}
\operatorname{ch} \beta\left(\frac{L}{2}-x_{0}\right) / \operatorname{ch} \beta \frac{L}{2} \\
\operatorname{sh} \beta\left(L-x_{0}\right) / \operatorname{sh} \beta L
\end{array}\right]
\end{aligned}
$$

is the voltage response-function in SD and SG circuits.

In the framework of such a model the spectral densities of current and voltage fluctuations at FET/HEMT terminals are described as

$$
S_{\xi \xi}(\omega)=\int_{0}^{L} n^{3 \mathrm{D}}\left|G_{\xi}\left(\omega, x_{0}\right)\right|^{2} S_{f f} \mathrm{~d} x_{0},
$$

where $G_{\xi}\left(\omega, x_{0}\right)$ is the spectral representation of the response function of the quantity $\xi=J, U$ to a local perturbation at $x=x_{0}$ induced by the Langevin force $f$.

\section{Main branching-induced effects}

\subsection{Time delay of response}

As an example, Fig. 1 presents the time response of currents at the source, drain and gate terminals, respectively, $\Delta J_{\mathrm{s}}, \Delta J_{\mathrm{d}}$ and $\Delta J_{\mathrm{g}}$, caused by a local perturbation appearing at time moment $t=0$ in point $x_{0}=0.05$ (just near to the source) and $0.45 \mu \mathrm{m}$ (near to the channel centre). When a perturbation occurs near to one of the channel boundaries (source or drain, see Fig. 1a), the current response at the perturbed terminal appears practically immediately, while at the opposite terminal it appears with a time delay of about 1 ps. In the case when the perturbation takes place near to the channel centre (see Fig. 1b), the delay of the current response appears at all the three terminals. Independently of the initial perturbation location, the temporal evolution of the $\Delta J_{\mathrm{g}}$ response tends to be synchronized with either the $\Delta J_{\mathrm{s}}$ or the $\Delta J_{\mathrm{d}}$, thus fulfilling the zero-sum rule, i.e. $\Delta J_{\mathrm{d}}-\Delta J_{\mathrm{s}}+\Delta J_{\mathrm{g}}=0$. Such a delay leads to the appearance of a series of resonant peaks, i.e. to an oscillatory behavior of noise spectra.

\subsection{Excitation of plasma waves}

Figure 2a presents the spectral density of current fluctuations in SD and SG circuits calculated at constant voltage operation $\left(\Delta U_{\mathrm{g}}=0, \Delta U_{\mathrm{d}}=0\right)$. Oscillations in the noise spectra are related with the resonant excitation 


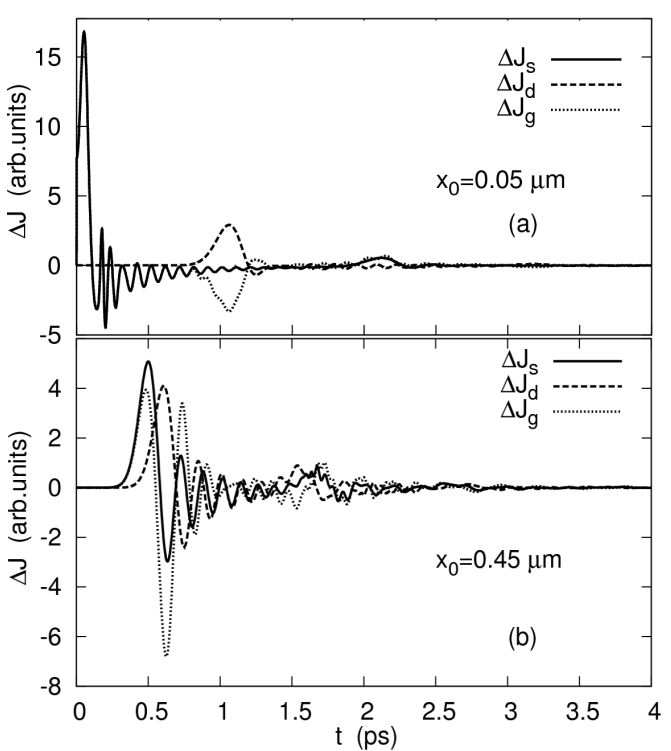

Fig. 1. Time response of currents respectively at source, drain, and gate terminals calculated by the hydrodynamic approach for a local perturbation introduced by the Langevin force at two different points $x_{0}=$ $0.05 \mu \mathrm{m}$ (a) and $x_{0}=0.45 \mu \mathrm{m}$ (b) of the channel. As boundary conditions we take $U_{\mathrm{g}}=0$ and $U_{\mathrm{sd}}=0$. Other parameters are: channel length $L=1000 \mathrm{~nm}$, thickness $\delta=15 \mathrm{~nm}$, channel to gate distance $d=15 \mathrm{~nm}$, donor concentration $N^{3 \mathrm{D}}=8 \times 10^{17} \mathrm{~cm}^{-3}$. Kinetic parameters of electrons in the channel correspond to $\mathrm{In}_{0.53} \mathrm{Ga}_{0.47} \mathrm{As}$ at room temperature, namely: $\nu=3 \times 10^{12} \mathrm{~s}^{-1}$ and $m^{*}=0.048 m_{0}$.

of spatial modes of plasma waves in the dielectric layer separating the channel from the gate. Here, the resonant frequencies can be determined by the denominator of the response functions of currents in Eq. (7), which finally gives [1]:

$$
\begin{gathered}
\omega_{\text {res }}^{i}(k)=\omega_{\mathrm{p}} \frac{k}{\sqrt{(\lambda L / \pi)^{2}+k^{2}}} \\
\left\{\begin{array}{l}
k=0,1,2, \ldots, i=\mathrm{SD}, \\
k=1,3,5, \ldots, i=\mathrm{SG} .
\end{array}\right.
\end{gathered}
$$

The full set of resonances $(k=0,1,2, \ldots)$ corresponding to the excitation of standing waves appears for fluctuations in the SD circuit only, while in the SG circuit one observes only odd spatial modes $(k=1,3,5, \ldots)$. As follows from Eq. (12), the spectrum of excited plasma modes exhibits an upper limit at $k \rightarrow \infty$ given by the 3D plasma frequency $\omega_{\mathrm{p}}$. Let us note that the widely used gradual channel approximation of the Poisson Eq. [3] (obtained when the second spatial derivative in equation (2) is omitted) gives an infinite equidistant spectrum of the Dyakonov-Shur plasma waves which corresponds to the limit $k \ll \lambda L / \pi$ in our Eq. (12).

\subsection{Interference of currents in the gate}

The absence of even modes in the spectrum of current fluctuations in the SG circuit (see Eq. (12) and Fig. 2a)

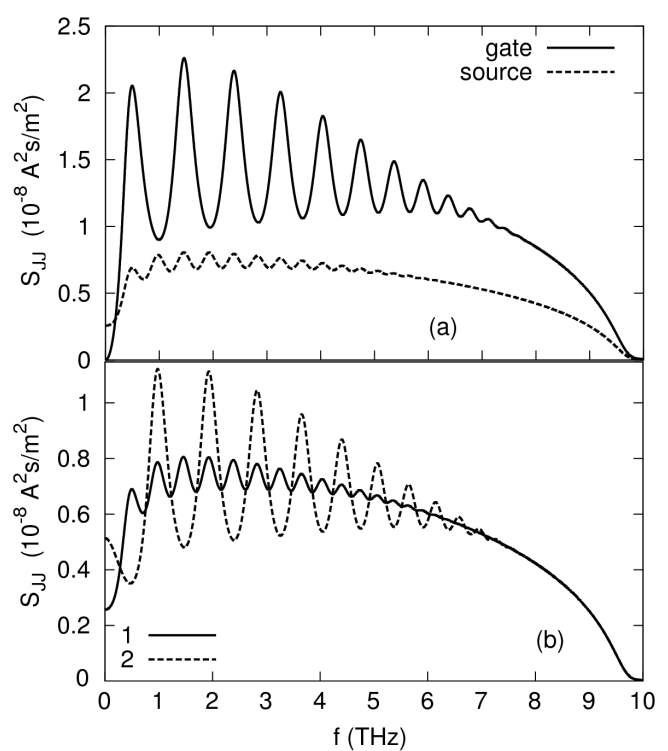

Fig. 2. Spectral density of current fluctuations: (a) in SD and SG circuits calculated with $U_{\mathrm{sd}}=0$ and $U_{\mathrm{g}}=0$, and $(\mathrm{b})$ in an SD circuit for a short $\left(\Delta U_{\mathrm{g}}=0\right)$ and open $\left(\Delta J_{\mathrm{g}}=0\right) \mathrm{SG}$ external circuit (curves 1 and 2 , respectively).

is related to the interference of local channel-to-gate currents, since, due to continuous branching, their spatial sum gives a zero value to the SG current for even modes. Indeed, a non-zero contribution is given only by those spatially excited modes whose symmetry center coincides with the center of the gate. Thus, even if a mode is excited its contribution can be "invisible" due to symmetry restrictions for certain circuits.

\subsection{Influence of embedding circuits on the noise spectrum}

Another situation can be realized when, due to some symmetry restrictions (introduced, for example, by embedding circuits), a mode cannot be excited at all. Let us demonstrate that a change of the operation regime of one of the adjoint circuit (e.g. SG or SD) leads to a corresponding change of the current fluctuation spectrum. Figure 2b reports noise spectra of current fluctuations $S_{j j}$ in the SD circuit in two cases, namely: when the $\mathrm{SG}$ circuit is short $\left(\Delta U_{\mathrm{g}}=0\right)$, or open $\left(\Delta J_{\mathrm{g}}=0\right)$. As follows from Fig. 2b, under the transition from the former case to the latter one, the fluctuation spectrum $S_{j j}$ loses the contribution of the odd harmonics of plasma excitations, thus only the even $(k=2,4,6, \ldots)$ harmonic contribution remains in the spectrum. Indeed, when the SG circuit is open, so that $\Delta J_{\mathrm{g}}=0$, spatially-different partial contributions of the channel-to-gate current must fully compensate each other to provide zero current at the gate. This condition can be satisfied only for even modes with the inversion centre in the channel center. Thus, such an influence of the operation mode of one of adjoint circuits on the noise spectrum in the other one 
destroys the property of the Thevenin and Norton generators to describe the intrinsic noise in embedding circuits independently of their properties.

\subsection{Excess noise due to plasma excitations}

Figure 3a demonstrates the appearance of an excess noise in the current fluctuation spectra caused by the excitation of plasma waves. For the ungated channel, $d \rightarrow \infty$, we obtain the usual Lorentzian spectrum. With the decrease of the channel-to-gate distance $d$ there appears an excess noise at frequencies below the 3D plasma value $\left(f=\omega_{\mathrm{p}} / 2 \pi \approx 10 \mathrm{THz}\right)$. With the decrease of $d$, the frequency region involved into the excess noise expands into the low-frequency region. This is accompanied by the growth of the noise intensity inside the excess noise region. Figure $3 \mathrm{~b}$ shows the integral intensity of current noise in an SG circuit, $\overline{\Delta j^{2}}=\int_{0}^{\infty} S_{j j}(\omega) \mathrm{d} \omega$, as a function of dimensionless parameter $\gamma=L \lambda \sim L / \sqrt{\delta d}$
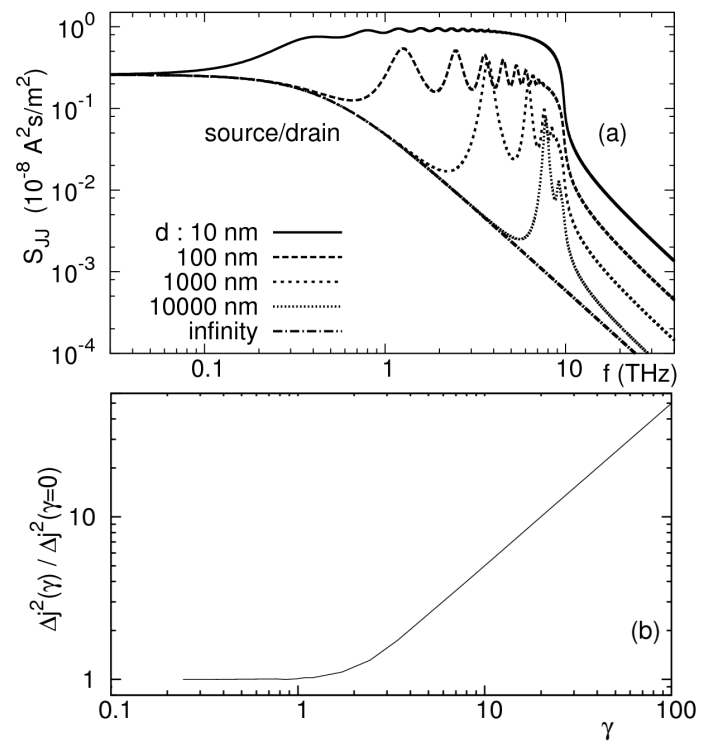

Fig. 3. (a) Spectral density of current fluctuations in an SD external circuit calculated with $U_{\text {sd }}=0$ and $U_{\mathrm{g}}=0$ at different values of the distance $d$ between the channel and the gate, and (b) integral intensity of current noise in an SD circuit vs. the dimensionless parameter $\gamma=L \lambda \sim L / \sqrt{\delta d}$.

determined by ratio between the transverse channel-to-gate capacitance and the longitudinal capacitance of the channel-under-gate region. As follows from Fig. 3a, when $\gamma<1$ (plasma excitations are absent) $\overline{\Delta j^{2}}$ keeps a constant value. When $\gamma>1$ there takes place an exponential growth of $\overline{\Delta j^{2}}$ which, in accordance with Fig. 3a, is correlated with the increase of the number of plasma modes excited between the channel and the gate.

\section{Conclusions}

The considered above analytical model of the diffusion noise in FET/HEMT channels allows us to conclude: as a result of the continuous branching of the total current under the gate-governed transistor channel there appears a new phenomenon, namely: a set of spatial modes of 2D-plasma waves excited at the frequencies given by Eq. (12). Such a conclusion is based on the following consequences of the model: (i) These 2D-plasma waves are excited in the frequency region $\omega<\omega_{\mathrm{p}}^{3 \mathrm{D}}$ which is well below of the threshold of the 3D-plasma oscillations where excitation of usual 3D electromagnetic waves is forbidden by the dispersion law. Moreover, their appearance cannot be interpreted as a transformation of 3D eigenmodes caused by the changing in the boundary conditions only. (ii) The integral intensity of diffusion noise in FET/ HEMT channels grows proportionally with increase of a number of excited 2D plasma waves (see Fig. 3).

\section{Acknowledgments}

This work is supported by grant No. MIP-87/2010 of Lithuanian Science Council.

\section{References}

[1] P. Shiktorov, L. Varani, G. Sabatini, H. Marinchio, L. Reggiani, J. Stat. Mech. 01, 01047 (2009).

[2] P. Shiktorov, E. Starikov, V. Gružinskis, L. Varani, L. Reggiani, AIP Conf. Proc. 1129, 179 (2009).

[3] P. Shiktorov, E. Starikov, V. Gružinskis, S. Pérez, T.González, L. Reggiani, L. Varani, J.C. Vaissière, Phys. Rev. B 67, 165201 (2003).

[4] M. Dyakonov, M. Shur, IEEE Trans. Electron Dev. 43, 1640 (1996).

[5] J.-F. Millithaler, L. Reggiani, J. Pousset, L. Varani, C. Palermo, W. Knap, J. Mateos, T. Gonzalez, S. Perez, D. Pardo, Appl. Phys. Lett. 92, 042113 (2008). 